\title{
Influence of the magnetic field on microorganisms in the oral cavity
}

\author{
Snezana BRKOVIC'1, Srdjan POSTIC ${ }^{1}$, Dragan ILIC² \\ 1- Clinic for Prosthodontics, School of Dental Medicine, Rankeova 4, University of Belgrade, Belgrade, Serbia \\ 2- Clinic for Endodontics, School of Dental Medicine, Rankeova 4, University of Belgrade, Belgrade, Serbia
}

Corresponding address: Snezana Brkovic - Clinic for Prosthodontics, School of Dental Medicine, Rankeova 4, University of Belgrade - Beograd - Serbia Phone: +381 1131933 07/0631302963 - e-mail: brkovics@vektor.net

Submitted: July 11, 2014 - Modification: October 27, 2014 - Accepted: October 30, 2014

\section{ABSTRACT}

\begin{abstract}
Since the beginning of their lives, all living organisms are exposed to the influence of geomagnetic fields. Objectives: With respect to the positive effects that magnetic fields have on human tissues, especially the bactericidal effect, this investigation aimed to assess their influence on the reduction of oral microorganisms. Material and Methods: In order to obtain adequate specimens of dental plaque deposit, microbes such as Streptococcus parasanguinis, Staphylococcus epidermidis, Rhodococcus equi and Candida albicans were isolated from the human mouth. To establish the intensity of microbial growth on the basis of the modified optical density (OD) of agar turbidimetry assay, microbial count and spectrophotometry were applied. The study was carried out with two microbial concentrations ( 1 and $10 \mathrm{CFU} / \mathrm{ml}$ ) after periods of incubation of 24 and $48 \mathrm{~h}$ and using micromagnets. Results: A positive effect of the magnetic field, resulting in the reduction of dental plaque microbes in vitro, was found. In the first 24 hours of exposure to the magnetic field, the number of all isolated microbes was significantly reduced. The most potent influence of magnets and the most intensified reduction after 24 hours were evident in Candida albicans colonies. The decrease in the influence of magnets on microbes in vitro was also detected. Conclusions: Magnets reduce the number of microbes and might be recommended as a supplement in therapy for reduced periodontal tissues. This is important because periodontal tissues that are in good conditions provide prolonged support to the oral tissues under partial and supradental denture.
\end{abstract}

Keywords: Microbes. Oral cavity. Magnetic field. Overdenture. Candida albicans. Streptococcus parasanguinis.

\section{INTRODUCTION}

Living creatures have been exposed to the influence of geomagnetic and electric fields since the beginning of their lives on Earth. There is a lot of evidence and studies about magnet applications in medicine $8,12,13,14,23$. One of them, using lab rats, has been performed in the conditions of 60 times the power of the geomagnetic field. Growth on these animals has been reported on the $5^{\text {th }}, 13^{\text {th }}$ and $26^{\text {th }}$ days of the postnatal period, prior to euthanasia. The animal mortality increased by $30 \%$ when compared with control groups. Moreover, it has been observed that young animals showed a state of hyper mobility which has been followed by paralysis. Yellow smear have been found on their livers, and cirrhosis was frequent among rats ${ }^{22}$. According to the information learned, living organisms create a reaction of effectors that generates changes in the inner electromagnetic field. These changes have been modified by periodical transformations, occasionally related to the non-periodic fluctuations of the outer electromagnetic field of the earth ${ }^{20}$.

In experiments in which $E$. coli has been added to the water running between two magnetic polarities with a speed of $0.5 \mathrm{~L}$ per hour and intensity of magnetic field of $39.9 \mathrm{KA} / \mathrm{m}$, a number of bacteria have been reduced from $10^{5}$ to 0 . This has particularly been observed in occasions when water was heated up to $60^{\circ} \mathrm{C}$. When water was moderately heated, without the influence of the magnetic field, the antibacterial effects were not 
present $^{3,4,22}$.

In favor of antibacterial effect, it could be stated that in the case of the laboratory animals with infected fractured bones, the magnetic field increased the effect of antibiotics with reduction of posttraumatic oedema and acceleration of fracture healing $11,20,22$.

Electromagnetic fields in the region of the edentulous ridge significantly decrease and slow down the residual ridge reduction ( $R R R)^{22}$.

$A$ relation between influence of the magnetic field and reduction of microbes in natural water has also been evidenced. Microbes act in a "magneticsensitive" manner. This means that microbes have been oriented and distributed along force lines within environments rich in ferric and magnetic oxides. This orientation with transition of microbes has been designated "magnetic-vowing", which means microbe orientation to the magnetic field. Magnet-sensitive microbes have been evaluated in early 1970s. During changes of direction within the field itself, microbes turn around to reach the direction of the magnetic field. This phenomenon has been added to a number of studies on the various microbes with two common attributes, all of them were anaerobic and their magnetic osmolarity due to the intracytoplasmic structure of $\mathrm{Fe}_{3} \mathrm{O}_{4}$.

A number of studies report the influence of the electromagnetic field on the inhibition of growth of microbes and fungi, as well as slowing down their multiplication ${ }^{19}$.

There are controversial opinions about microbe growth. For example, the results of experiments with the well-studied Vibrio campbellii strain BB120 (originally classified as Vibrio harveyi) and derivative mutants unable to synthesize autoinducers suggest that the effects of magnetic fields on quorum sensing may be mediated by AI-2, the interspecies quorum sensing signal molecule ${ }^{21}$. Kohno, et al. ${ }^{15}$ (2000) has stated that growth under static magnetic field depends on the experimental conditions.

In 1992 Grossmann, et al. investigated the effect of magnetic fields on the microbes Escherichia coli (E. coli) and Staphylococcus aureus (Sta. aureus) ${ }^{16}$. Microorganisms were exposed to a static magnetic field of 0.5-4 T (Tesla unit) power for 30-120 minutes. No significant effects on the growth and biochemical activity of microbes were noted, as with antibiotics.

Many researchers have advocated the fact that magnetic fields inactivate only pathogenic microbes ${ }^{1,2,3}$.

\section{MATERIAL AND METHODS}

The materials used in this study are samples of dental plaque deposits under in vitro conditions originated from the surfaces of the teeth of patients of the Clinic for Prosthodontics - Belgrade School of Dental Medicine and Belgrade Military Medical Academy. The magnetic field has been obtained from AKMA micromagnet pieces created by Institute Mihajlo Pupin, Belgrade, Serbia, and certified by the Military Medical Academy, Belgrade, Serbia, certification number $1600-2 / 83^{12}$.

The magnets used are based on a ceramic barium ferrite $(\mathrm{BaF})$. The magnets were planconvex discs with a diameter of $3 \mathrm{~mm}$, height of $1.4 \mathrm{~mm}$ and the radius of action of $5-8 \mathrm{~mm}$. Considering all of that determined data, we were able to accurately apply it to the parts of dentures where we wanted to achieve a homogeneous magnetic field. Since the magnet is fixed on the overdenture base in the problem area, as the one treated zone that does not change the oscillation of the field in the tissue and which is applied to this, the treated field was considered the homogeneous one.

The magnetic field was static. The intensity on the surface of the magnet was $60 \mathrm{mT}$. The power of the magnet decreases as the distance from the magnet in the proportion $1 / \mathrm{r}^{3}$ up to $1 \mathrm{mT}$ when there is no influence on the tissue metabolism.

According to the World Health Organization (WHO) standards, this field was classified as "safe" for human tissue treatments with no consequences, for an indefinite period, i.e., more consecutive months.

The advantages of static magnetic fields consist in the fact that the user clearly knows how the field continuously treated some of the tissue. Variable magnetic fields are very questionable in terms of frequency of the field, field strength, and diameter and depth of field effects. Static magnetic fields are easy to control and it is possible to use them with no restrictions, in continuum. Adversely, variable magnetic fields are restricted to the laboratory environment and the patients must be constantly charged to current, which makes them non-appropriate for everyday dental use. In our experiment, magnet discs were oriented on the overdenture base in a way that the plain surface of the disc (south pole) was oriented inward and the convex surface (north pole) to the outward - to the gingival tissue - therapeutic pole.

\section{Material used in microbiological in vitro researches}

It was necessary to obtain adequate samples (smears of dental deposits or specimens of dental plaque) wherefrom certain microbes have been isolated for the scope of the in vitro investigation into the influence of AKMA micromagnets upon possible reduction of the number of bacteria isolated in the human mouth, in the microbiology and mycology laboratory (Department of Microbiology - 
School of Veterinary Medicine, Belgrade University). Microorganisms identified as Streptococcus parasanguinis, Sta. epidermidis and Rhodococcus equi strains have been isolated from pure cultures. Preservation of isolated strains was enabled by an assay including the reference strain of $C$. albicans ATCC 23344 (Becton Dickinson). The selection of bacterial species intended to refer to an assay based upon bibliographic data proving that Str. mutans and C. albicans play a crucial role due to their ability to adhere onto materials (teeth, acrylic) and simultaneously colonize epithelized surfaces. The rest of the referred species also accelerate plaque formation but much less than the previous ones. Rhodococcus equi was selected for being an opportunistic pathogenic member of the group of the so-called coryneform bacterial culture that is well-known for its influence on plaque formation. Blood agar was enriched by adding $6 \%$ of sterile sheep blood. Nutritional substrate was used for isolation of the referred bacterial strains (BioLab). Lyophilized rabbit plasma diluted at $1: 5$ ratio (Becton Dickinson), purpur agar (Oxoid), DNA agar (Becton Dickinson), "Staph-ident" system for latex agglutination tests (BioMerieux), SlidexStrepto (BioMerieux) system for latex agglutination tests and automatic identification system BD Crystal Gram Positive ID (Becton Dickinson) were used for standardization, i.e., for final strain identification. Semi-liquid agar media of $0.5 \%$ and $\mathrm{BH}$ I agar (Merck) with the addition of $30 \%$ glycerol (ICN) were used for preservation of referred bacterial strains. Dextrose agar (BioLife) and Sabouraud agar (BioLab) were applied for assaying the influence of the magnetic field upon bacterial number reduction. Sterile physiological solution was used for the scope of preparation of inoculums of assayed bacterial strains. Initial inoculums density of $1 \times 10^{8}$ bacteria/ $\mathrm{ml}$ was achieved by comparison of the dilution of a 0.5 McFarland standard solution with a Becton Dickinson scale.

\section{Material for in vivo experiment}

This material was obtained from 38 men and women with similar oral status and oral treatment: conventional upper denture and lower overdenture on a small number of remaining teeth.

An accumulation of dental plaque under in vivo conditions was found in the remaining teeth under overdentures. Firstly, the samples of dental plaque from the determined surfaces were taken before incorporation of magnets in the denture base on the seventh day. Patients were asked to follow oral a hygienic regime without the use of tooth brushes and to rinse their mouths using only water. These microbes have been smeared in vivo on microscope slides using two or three drops of distilled water.
A) Methods of in vitro examination into the influence of AKMA micromagnets on the dental plaque bacteria

The precondition for carrying out this in vitro investigation enabled the application of microbiological investigation methods which necessitated the isolation and identification of evaluated bacteria to analyze the influence of AKMA micromagnets-related magnetic fields upon reduction of the number of microbes.

\section{Isolation and identification of examined bacterial strains \\ Isolation and identification of examined bacterial} strains was achieved with the application of conventional microbiological methods. Finally, affirmative identification has been carried out by the application of automatic identification systems. Target isolation of Sta. epidermidis, a subgroup of Str. milleri (Str. sanguinis, Streptococcus parasanguinis, Str. intermedius), Str. mutans, Rhodococcus equi, Prevotella species, C. albicans etc. was completed. Those microbes substantially hindered work activities and prolonged evaluation time since all of the abovementioned strains, of significance in dental plaque formation, are present in small numbers and were outnumbered many times by present fast-growing bacteria. Nonhaemolytic coagulase-negative staphylococci and DNA belonging to Str. epidermidis species were selected for further investigation.

When referring to streptococci, further investigations were only performed using alphahaemolytic specimens. The circumstances were additionally hindered by the fact that all Streptococcus specimens are responsible for dental plaque formation and belong to the Alpha viridans haemolytic streptococci group that includes numerous opportunistic pathogen strains continually present in the mouth (Str. pneumoniae, Enterococcus specimens). All abovementioned bacterial strains are facultative anaerobe, unlike Str. Mutans, which is necessarily anaerobe; it means that all referred bacterial strains, resulting from processed samples, were growing quite well in anaerobic conditions of cultivation that practically prevented isolation and identification of the required Str. mutans. The reason to apply conventional bacteriological methods is due to the fact that those species are justifiably responsible for dental plaque formation. Afterwards, after applying the BD Crystal Gram Positive ID set, the referred strains were standardized up to the specimen.

Regarding Rhodococcus equi identification, CAMP was performed on blood agar employing Staphylococcus aureus ATCC 6538P and pigmentation of colonies of this bacterial specimen. 
Examination of the influence of AKMA micromagnets field on bacteria reduction

Turbidimetric assay has been applied for this type of investigation, which involves spectrophotometric measuring, i.e., indirect determination of the growth intensity of seeded microorganism strains on the basis of the modified optical density (OD) of the agar.

Sterile dextrose agar and Sabouraud dextrose agar were standardized as the "zero" value of OD on spectrophotometer. Reading was made at a 546 $\mathrm{nm}$ wavelength (recommended value). Bacterial cultures have been seeded in Erlen-Mayer flasks (EM) with $100 \mathrm{ml}$ dextrose agar, as well as the $C$. albicans yeast strain.

The effect of AKMA magnets was evaluated in agar media with different numbers of bacteria and yeasts.

The $10^{3}$ microbes were totally inoculated in the first series into $100 \mathrm{ml}$ of nutritional agar media. Inoculation was done in $1 \mathrm{ml}$ of previously prepared suspension containing the evaluated strains in a $10^{3}$ microbial density $(1.000 \mathrm{CFU} / \mathrm{ml})$. Thus, the initial count of agar-contained microbes amounted to $10 \mathrm{CFU} / \mathrm{ml}$. The inoculated agars with and without magnetic influence had the same number of microbes.

In the second series, a hundred bacteria were inoculated in agars containing magnets (inoculation of $1 \mathrm{ml}$ of previously prepared suspension of the evaluated microbes in a $100 / \mathrm{ml}$ density -100 CFU/ $\mathrm{ml})$. Thus, the initial count of microbes in agar amounted to $1 \mathrm{CFU} / \mathrm{ml}$. Inoculated agars without AKMA magnets have been arranged for comparison.

The referred densities of microbe suspensions have been obtained by the double dilution of previously prepared initial suspensions of $100 \mathrm{CFU} /$ $\mathrm{ml}$ bacterial/yeast density. Agars containing the referred number of inoculated microbes did not show different OD values on the spectrophotometer in comparison to sterile agars serving as zero standards. Agars that contained initial bacterial count of 1 and $10 \mathrm{CFU} / \mathrm{ml}$ were "clouded" and spectrophotometrically recorded as a modification of the OD value in comparison to the standard. That is why the initial values of inoculum density of the evaluated bacteria could amount up to 10 $\mathrm{CFU} / \mathrm{ml}$. Incubation has been set at $37^{\circ} \mathrm{C}$ and spectrophotometric readings have been done after 24 and 48 hour periods.

B) In vivo methods of investigation on the influence of AKMA micromagnets on dental plaque bacteria

The dental plaque samples were initially taken from the areas already determined in the in vivo conditions. Plaque accumulation was monitored on the remaining teeth at the experimental and at the control sides. The material collected from the teeth (plaque deposits) was not cultivated, but prepared directly onto microscopic slides. The smeared plaque material was prepared by adding of 2-3 drops of distilled water, which were subsequently dried in the air. Afterwards, the microscope slide was passed three times through a flame to fix the sample. Later on, the samples have been colored by Gram stain. Their identification was completed by means of a microscope with homogenous immersion. The total bacterial number was recorded in three visible fields on the basis of their respective morphological-tintorial characteristics within each observation period on individual samples.

After a 3-month screening, the patients were told to behave the same way as prior to the inclusion in the study, i.e., they bore common dentures with inserted magnets.

Regarding microbe strains, it was not possible to achieve standardization of the prevalence of some of them in dental plaque.

The ethics committee certificate was issued by the School of Dental Medicine, Belgrade, Serbia (certificate number $572 / 1$ issued on April $14^{\text {th }}$, 2006).

\section{RESULTS}

\section{Statistics}

The results of measurements of OD in experimental groups of microbes have been calculated as average values \pm SD (standard deviation). Student's t test has been used for comparison among the groups with a statistically significance level of 0.05 .

Results of microbiologic in vitro investigations

\section{A) Test results of series with an inoculum density of $1 \mathrm{CFU} / \mathrm{ml}$ \\ Based upon the OD values obtained by spectrophotometer reading, it could be observed that AKMA magnets influence the growth reduction of all investigated bacteria and yeast under in vitro conditions. OD values of inoculated agars with and without AKMA magnets are both shown in the figures.}

Figure 1 data point out to the reduced number of all investigated microbes following 24 hours of AKMA magnet effect. The greatest magnet influence, i.e., the most severe microbe reduction after $24 \mathrm{~h}$ of incubation, was noted in the $C$. albicans yeast strain. The OD values in Sabouraud dextrose agar without magnets were 8.4 times higher than those of the agar with magnet action. Similarly, the intensity of microbial growth in dextrose agar with Rhodococcus equi strain by magnet influence 
was 4.27 times lower in comparison to magnetfree agar.

AKMA magnets showed the least influence on microbial reduction in the Str. parasanguinis strain, which is noted in the lowest difference in the OD values obtained. There was only 1.28 times more intense growth in magnet-free agar than in magnet-containing agar. Figure 1 data indicate that the OD values of agar after $48 \mathrm{~h}$ upon incubation with or without magnets turned out to be very close themselves, i.e., AKMA magnets reduced the studied bacteria/yeast strains much less significantly in the second day of incubation. The $O D$ values of reference $C$. albicans strain in magnetcontaining agar increased 8.9 times following $24 \mathrm{~h}$ incubation (Figure 1).

Regardless of the significant increase of $O D$ values in magnet-containing agar following $48 \mathrm{~h}$ of incubation, the value of OD in AKMA-magnet-free agar was only 1.07 times higher in comparison with the values of magnet-containing agar. It is possible to conclude that magnets significantly influence the reduction of the number of $C$. albicans even after $48 \mathrm{~h}$ (Figure 1).

Rhodococcus equi behaves similarly. Micromagnets influenced their reduction even 48 $\mathrm{h}$ later, while the recorded values of OD of magnetfree agar amounted to 0.649 (only 1.126 times greater than the values of magnet-containing agar samples).

As for Str. parasanguinis, the OD values were achieved through an increased reduction of the total number of bacteria under the influence of AKMA magnet. That was noted on the second day of incubation. The OD values of the agar only increased to 1.01 , i.e., 0.007 times, calculated on the basis of the OD values obtained with magnets throughout the first 24 hours. In other words, streptococci almost failed to multiply in dextrose magnet-containing agar in the course of the second day of incubation.

\section{B) Test results for the series with inoculums density of $10 \mathrm{CFU} / \mathrm{ml}$}

On the basis of the obtained OD values having been read by the spectrophotometer it may be noted that AKMA micromagnets influence the reduction of the number of all tested bacterial and yeast strains under in vitro conditions. The resulting OD values of inoculated agars with and without AKMA magnets after $24 \mathrm{~h}$ and $48 \mathrm{~h}$ are shown numerically in Figure 2.

A
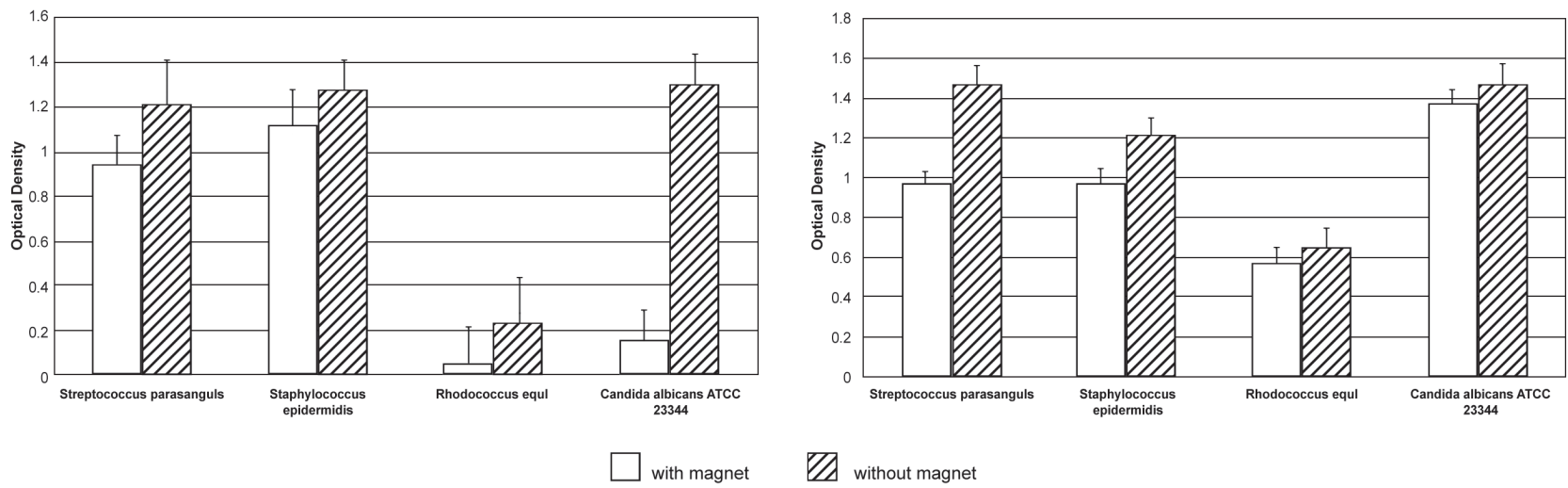

Figure 1- Optical density values upon 24 and $48 \mathrm{~h}$ of incubation with a inoculum density of $1 \mathrm{CFU} / \mathrm{ml}$

A
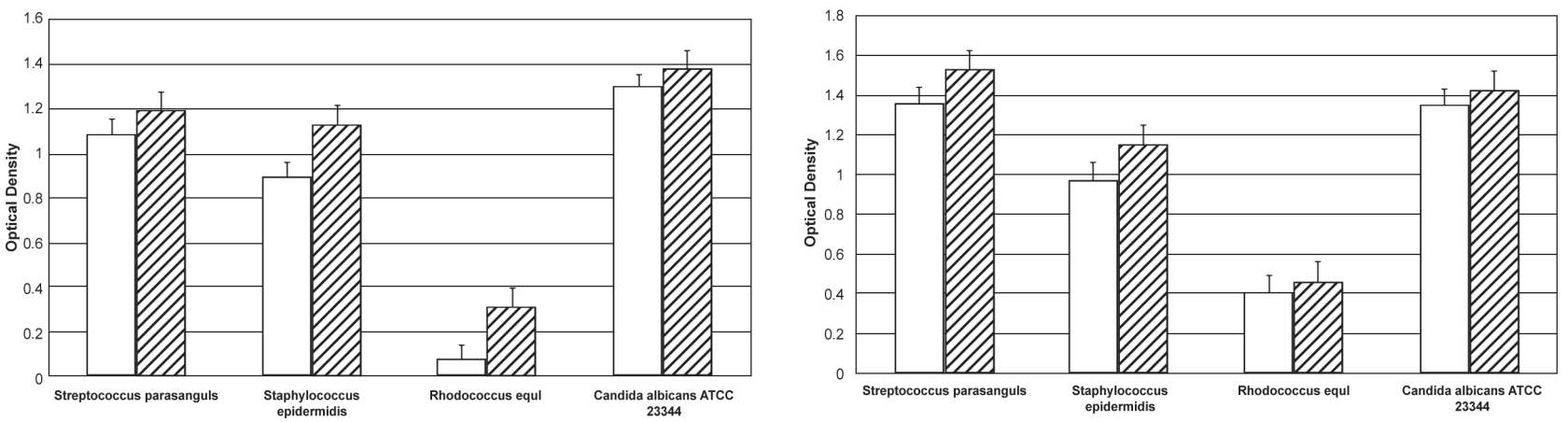

with magnet

Figure 2- Optical density values upon 24 and $48 \mathrm{~h}$ of incubation with a inoculum density of $10 \mathrm{CFU} / \mathrm{ml}$ 


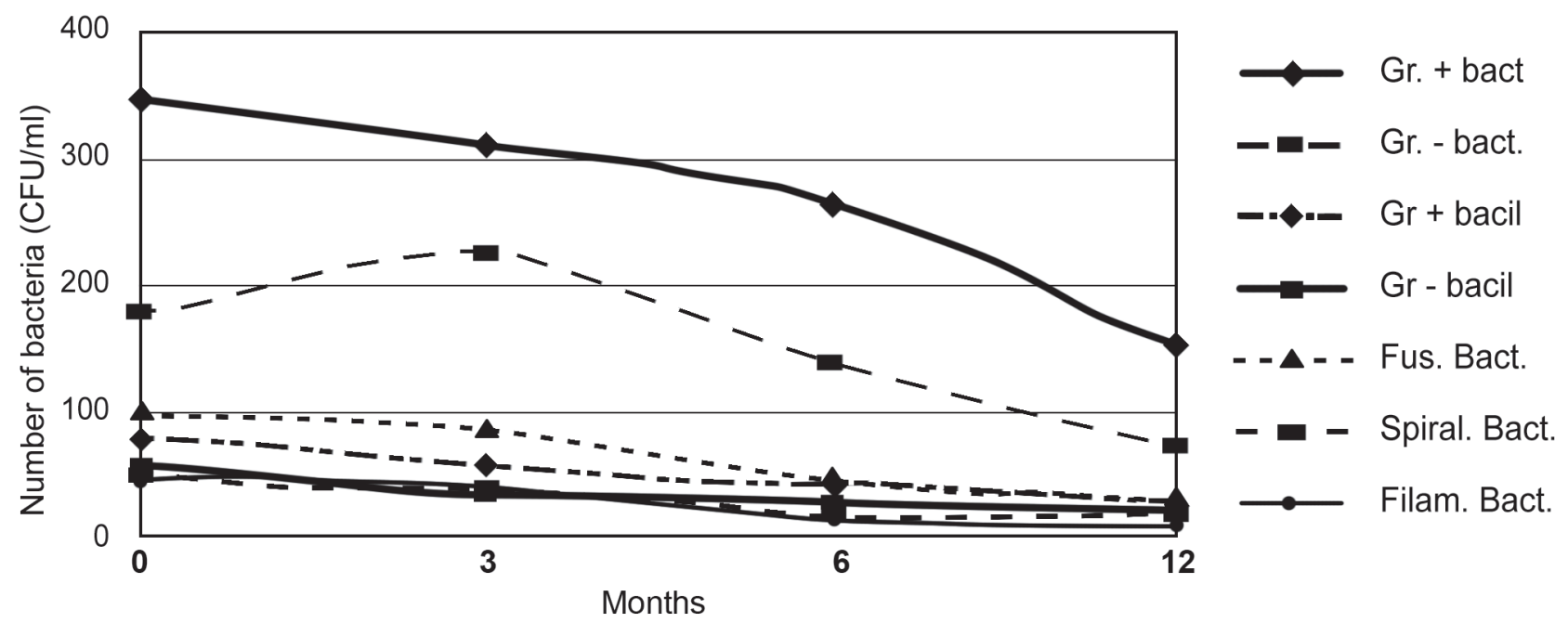

Figure 3- Average number of bacteria in dental plaque inside visible field in the defined observation periods

\section{Results of microbiologic in vivo investigations}

Results of in vivo investigation and analysis were assessed for every subject (patient). The distribution of all studied microbes is shown in Figure 3 , in which the decrease in the number of all studied plaque microbes is confirmed. There were no statistical significant differences between microbial counts because more precise evaluation was conducted within observation periods $(0,3,6$ and 12 months) (Figure 3).

\section{DISCUSSION}

The turbidimetry method has been widely discussed in the pharmacopeia of European countries, as well as the Yugoslavian pharmacopeia, with regard to application of bacteria to the nutrient media and antibiotic effect. On the basis of our study, $O D$ values obtained by the spectrophotometer and the degree of reduction of microbial growth could be also applied to the exact influence of antibiotics on microbes. In our study, magnets were used instead of antibiotics, with a specific method of application adapted to the purpose of the study. The modifications concerned the type and the amount of nutrient media as well as the initial number of microbes. Dextrose nutrient media was favored because it is completely clear when sterile, showing even minimal discoloration and opalescence.

Under in vitro conditions, AKMA micromagnets have shown a certain influence on microbial decrease in our study, but in the course of time this influence weakened and the number of microbes began to rise. Since the initial numbers of microbes were $10 \mathrm{CFU} / \mathrm{ml}$ and $1 \mathrm{CFU} / \mathrm{ml}$, and considering numerical OD values in the amount of 0.100 0.120 as related to the total number of $1-2 \times 10^{8} /$ $\mathrm{ml}$ of microorganisms, it could be presumed that the increase in the total number of microbes was evident after 24 hours. Some authors showed that magnets act less effectively under in vitro conditions, i.e., only slightly reducing the number of microbes ${ }^{19}$.

Since the in vitro reduction of microbial growth by magnetic field was confirmed, the next task of our study was to verify their reduction in the oral cavity of the patients (in vivo). For that purpose, the safety of application of magnets inside the mouth had been assessed in our pilot study ${ }^{9}$, in which it was determined that static magnets (of $\mathrm{BaF}$ content) do not expose the oral environment to corrosive effects, contrary to the findings of other authors ${ }^{9,16,17}$.

Specifically, for in vitro conditions, magnets influence the reduction of the number of microorganisms, but in the course of time, which could be seen in the obtained results, the influence is decreased and the number of microbes increases significantly again. The initial numbers of microbes in this assessment were $10 \mathrm{CFU} / \mathrm{ml}$ and $1 \mathrm{CFU} /$ $\mathrm{ml}$. Knowing that the $O D$ value of inoculated agar or other media of $0.100-0.120$ corresponds to a total number of microbes of $1-2 \times 10^{8} / \mathrm{ml}$, it could be concluded that after the initial 24 hours of incubation in agar with magnets, there was a significant increase in the total number of microbes, despite the evident influence of magnets on its reduction compared with agar without magnets. In other words, it is not the same to assess whether magnets have effects on 100 microbes or on $10^{6}$ microbes in $1 \mathrm{ml}$ of agar.

On the basis of the obtained results, it could be discussed how magnets affected the reduction in the number of microbes under in vitro conditions if the initial number was not great. When the total number of microbes is massively multiplied, magnets express less influence on their growth and differentiation. For in vivo, it is a prerequisite that the initial number of microbes is always at a low 
level, but when magnet has to be inserted into the dentures, it could continually influence the reduction in the number of microbes. Consequently, the detrimental influence of the low level of microbes is to be expected in the oral cavity. It could be speculated that magnet discs are more effective in the in vivo then in the in vitro experiments due to the oxygen level.

The obtained results obtained by the statistical method of ANOVA showed that magnets inserted into dentures provoke a significant reduction in the number of all detected microbes in dental plaque $(p<0.05)$. The $p$ values for only spiral microbes were at the low level of statistical significance $(P=0.05)$.

The effect of magnetic field is still controversial in literature data. Potenza, et al. ${ }^{17}$ (2004) stated that the magnetic field improves the $E$. coli proliferation, although Talà, et al. ${ }^{21}$ (2014) noted no differences in proliferation for a $V$. harveyi related strain.

Our in vitro investigation confirmed the effects of magnets on the reduction in the number of microbes. Additionally, in vivo investigation showed that for identical samples and without conducting the regime of oral and teeth hygiene, as well as for correct oral-hygienic procedures, a reduction in the number of microbes occurred. In both conditions, reduction in the number of microbes in staining smear isolated from dental plaque was documented at the beginning of the experiment. Literature data ${ }^{1,2,19}$ confirm those findings too, with a claim that magnets ${ }^{20,22}$ cannot replace the beneficial action of antibiotics but definitely could improve their bactericidal effects, as well as other healing methods $\mathrm{s}^{5,17,16}$. Apparently, magnets cannot substitute periodontal treatment (surgery) but might be an excellent supplement ${ }^{5}$.

Considering the effects of magnetic fields, it could be advocated that magnets have to be used as a supplement to conventional therapies ${ }^{3,18}$.

\section{CONCLUSION}

The positive effect of magnetic fields on the reduction in the number of dental plaque bacteria was confirmed in vitro. The influence of magnetic field was recognized in the first 24 hours of exposure. The count of all isolated microbes was significantly reduced. Over time, a decrease in the influence of magnets versus in vitro microflora density was noted. Beneficial influence of magnetic fields on the reduction of the number of dental plaque microbes was also proved in vivo. A reduction in the number of microbes was noted under overdentures containing inserted magnets in all observation periods (3, 6 and 12 months). Static magnetic fields applied in the area of the remaining teeth have beneficial effects on the alveolar bone and other periodontal tissues.

\section{ACKNOWLEDGEMENTS}

The author thanks Prof. Asanin Ruzica, specialist of microbiology at Belgrade University, School of Veterinary Medicine, for her part in this investigation.

\section{REFERENCES}

1- Abdel-Kader HM, Aref MI, Yousef SW. The biological effects of static magnetic field of commercial samarium-cobalt (SmCo5) orthodontic magnets on cultured Escherichia coli and staphylococci aureus. Int J Clin Dent. 2012;5(3):201-10.

2- Bajpai I, Saha N, Basu B. Moderate intensity static magnetic field has bactericidal effect on $E$. coli and $S$. epidermidis on sintered hydroxyapatite. J Biomed Mater Res B Appl Biomater. 2012;100(5):1206-17.

3- Brković-Popović S, Stamenković D, Stanisić-Sinobad D, Rakocević Z, Zelić O. The influence of continuous magnetic field on periodontal tissues under overdentures. Srp Arh Celok Lek. 2009;137(7-8):363-70.

4- Creanga DE, Poiata A, Morariu VV, Tupu P. Zero-magnetic field effect in pathogen bacteria. J Magn Magn Mater. 2004; 272:2442-4.

5- Darendeliler MA, Zea A, Shen G, Zoellner H. Effects of pulsed electromagnetic field vibration on tooth movement induced by magnetic and mechanical forces: a preliminary study. Aust Dental J. $2007 ; 52(4): 282-7$.

6- Esmekaya MA, Acar SI, Kiran F, Canseven AG, Osmanagaoglu $O$, Seyhan N. Effects of ELF magnetic field in combination with iron(III) chloride $(\mathrm{FeCl} 3)$ on cellular growth and surface morphology of Escherichia coli (E. coli). Appl Biochem Biotechnol. 2013;169(8):2341-9.

7- Faraj KA, Muhamad DA. Effect of high magnetic field on gram negative bacteria. Eur J Sci Res. 2012;74(2):240-3.

8- Feychting $M$. Health effects of static magnetic fields-a review of the epidemiological evidence. Prog Biophys Mol Biol. 2005;87(23):241-6.

9- Fojt L, Strasak L, Vetterl V, Smarda J. Comparison of the low-frequency magnetic field effects on bacteria Escherichia coli, Leclercia adecarboxylata and Staphylococcus aureus. Bioelectrochemistry. 2004;63(1-2):337-41.

10- Grosman Z, Kolár M, Tesaríková E. Effects of static magnetic fields on some pathogenic microorganisms. Acta Univ Palacki Olomuc Fac Med. 1992;134:7-9.

11- Hsu SH, Chang JC. The static magnetic field accelerates the osteogenic differentiation and mineralization of dental pulp cells. Cytotechnology. 2010;62(2):143-55.

12- IMP-PIEZOTEHNOLOGIJA DOO [homepage]. Subotica: NovaMedia; c2009 [cited 2014 July 7]. Available from: http:// novamedia.rs/showCategory/13203/mikromagneti-akma.

13- Janković BD, Marić D, Ranin J, Veljić J. Magnetic fields, brain and immunity: effect of magnetic field. Int J Neurosci. 1991;59(13):25-43.

14- Jovanovic-Nesic K, Eric-Jovicic M, Spector NH. Magnetics stimulation of the brain increase $\mathrm{Na}+, \mathrm{K}+$-ATPase activity decreased by injection of $\mathrm{AlCl}_{3}$ into nucleus basalis magnocellularis of rats. Int J Neurosci. 2006;116(6):681-95.

15- Kohno M, Yamazaki M, Kimura I, Wada M. Effect of static magnetic fields on bacteria: Streptococcus mutans, Staphylococcus aureus, and Escherichia coli. Pathophysiology. 2000;7(2):143-8. 16- Morrow AC, Dunstan RH, King BV, Roberts TK. Metabolic effects of static magnetic fields on Streptococcus pyogenes. Bioelectromagnetics. 2007;28(6):439-45.

17- Potenza L, Ubaldi L, De Sanctis R, De Bellis R, Cocchiarini L, Dachà $M$. Effects of a static magnetic field on cell growth and gene expression in Escherichia coli. Mutat Res. 2004;561(1-2):53-62. 
18- Steffensen B, Caffesse RG, Hanks CT, Avery JK, Wright N. Clinical effects of electromagnetic stimulation as an adjunct to periodontal therapy. J Periodontol. 1988;59(1):46-52.

19- Strasak L, Vetterl V, Fojt L. Effects of $50 \mathrm{~Hz}$ magnetic fields on the viability of different bacterial strains. Electromagn Biol Med. 2005;24(3):293-300.

20- Strasak L, Vetterl V, Smarda J. Effects of low-frequency magnetic fields on bacteria Escherichia coli. Bioelectrohemistry 2002; 55(1-2):161-4.

21- Talà A, Delle Side D, Buccolieri G, Tredici SM, Velardi L, Paladini $F$, et al. Exposure to static magnetic field stimulates quorum sensing circuit in luminescent Vibrio strains of the Harveyi clade. PLoS One. 2014;9(6):e100825.
22- Van der Kuij P, Vingerling PA, Sillevis Smitt PA, de Groot K, de Graaf J. Reducing residual ridge reduction. In: van Rens TJ, ed. Electric and electromagnetic stimulation of bone growth. New York: S. Karger; 1985. p. 98-105.

23- World Health Organization. Electromagnetic fields and public health [online]. March 2006. [cited 2014 July 7]. Available from: http://www.who.int/peh-emf/publications/facts/fs299/en/. 